\title{
IL TERREMOTO SARDO DEL 13 NOVEMBRE 1948
}

\author{
Francesco Peronaci
}

II 13 novembre 1948 alle $9^{\text {h }} 52^{\mathrm{m}}$ ca. T.M.G. fu avvertita in tutta la Sardegna settentrionale una scossa di terremoto che produsse danni agrli edifici situati nei paesi circostanti la zona di Tempio Pausania; particolarmente colpiti risultarono Aggius, Trinita, Badesi. Dal 13 novembre in poi vennero avvertite una ventina di repliche con intensità via via decrescente, e tra esse le più intense furono quelle del 16 alle $22^{\mathrm{h}} 55^{\mathrm{m}}$, del 21 alle $22^{\mathrm{h}}$, dell' 8 dicembre alle $4^{\mathrm{h}} 35^{\mathrm{m}}$ e alle $13^{\mathrm{h}} 15^{\mathrm{m}}$ tutte valutate sul $4^{\circ}-5^{\circ}$ della scala Mercalli.

Il periodo sismico produsse grande allarme sulla popolazione locale, tanto più che la Sardegna, pur essendo vaste zone di essa occupate da rocce terziarie di caraltere erutlivo, è da ritenersi terra pochissimo soggetla a fenomeni sismici. La storia della regione infatti, ci consente di rilevare un centro sismico nella parte meridionale dell'isola che dette origine a scuotimenti negli anni $1615,1771,1835$, 1855, mentre nella settentrionale si ebhe un solo terremoto nel giugno del 1870 la cui intensita i da valutarsi sul $5^{\circ}$ della scala Mercalli e il cui epicentro macrosismico i da porsi nella regione di Ittireddu. Naturalmente questi dati vengono riportati in hase alle notizie di carattere macrosismico in quanto, solo di recente, per iniziativa dell'I.N.G. è stata installata a Cuglieri una stazione sismica, che consentirà di seguire da vicino e con rigore scientifico la storia dell'isola.

Le registrazioni della rete italiana e degli Osservatori del centro Europa si riferiscono solo alla prima scossa; ne segue che il nostro studio sara limitato solamente ad essa. La raccolta dei dati macrosismici ì stata effettuata utilizzando oltre che del servizio macrosismico che fa capo all'I.N.G., anche delle notizie rentilmente fornite per la Corsica dal Bureau Central Séismologique Français nonché da osservazioni direllamente effeltuate sul posto dallo scrivente durante il periodo sismico in oggetto.

L'esame delle isosiste tracciate in fir. 1 mostra un andamento piuttosto regolare nella propagazione dell'energia, salvo nella zona di Giave e di Ittireddu ove l'irregolarità può giustificarsi col fatto che detta 
regione trovasi nella zona di saldatura tra terreni sedimentari e tranitici. Questa considerazione spiega come la scossa sia stata sentita di 4" a Bono, mentre a Florianas e a Sassari, notevolmente più vicine all'epicentro, essa non abhia superato il $3^{\circ}$; queste ultime infatti si trovano su terreni di natura sedimentaria i quali, pur esaltando le solle-

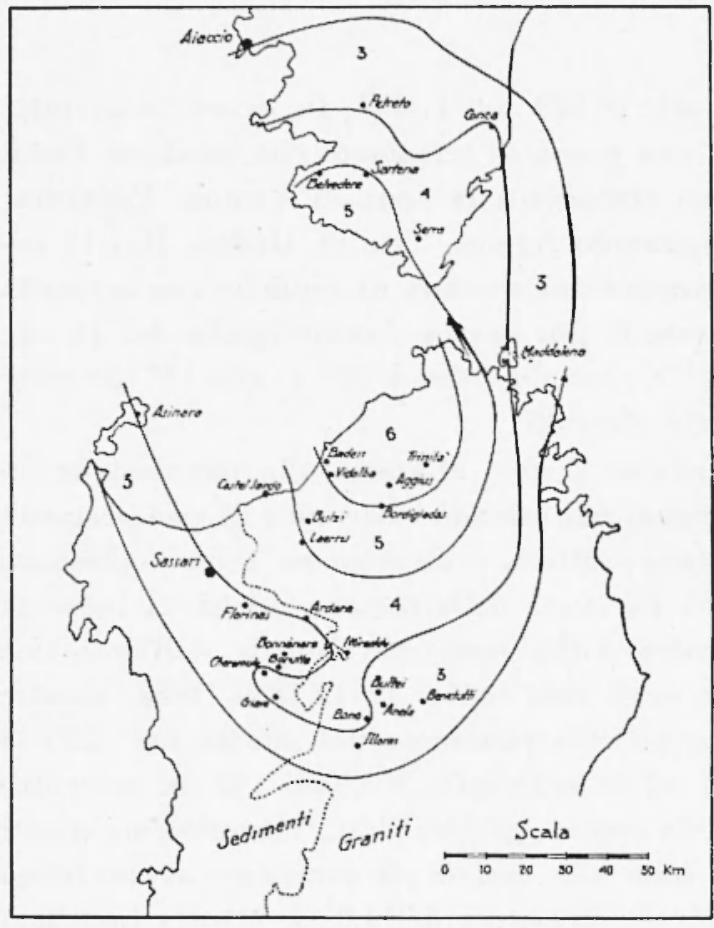

Fig. 1 citazioni all' ingresso della stratificazione, determinano successivamente un notevole assorbimento di energia per trasformazione in lavoro di attrito. Analogamente facciamo rilevare clue Monteggiani, che dista pochi chilometri da $\mathrm{Ba}$ desi non ha subito danni apprezzabili, es. sa infatti $\dot{e}$ situata immediatamente oltre la frattura geologica suddetta. Restano quindi ancora una volta confermate le considera. zioni fatte da Sieber e altri sismologhi, secondo le quali la costituzione del sottosuolo influisce in mo-

do essenziale nella determinazione dei danni provocati da una scossa, e come nella conseguente valutazione delle notizie macrosismiche non si possa prescindere dalla conoscenza geologica della regione.

In base alle registrazioni della stazione di Roma abbiamo ottenuto per la magnitudo 5; questo valore comporta un'energia liberata all'epicentro dell'ordine di $10^{20} \mathrm{erg}$, e confrontando questo dato con la scala Mercalli si ottiene che all'epicentro la scossa è di $7^{\circ}$. Tenendo conto che, come vedremo, l'epicentro è in mare in prossimità della costa, si ha una conferma delle notizie che pongono la massima intensità al $6^{\circ}$.

Una indagine eseguita sui danni agli edifici ci ha consentito di 
stabilire che essi presentano in genere le stesse caratteristiche: distacco di volte da muri di sostegno e gravi lesioni negli spigoli; data la modesta intensità della scossa, riteniamo cbe essì siano imputabili più che altro alle nome costruttive degli edifici stessi. Abluiamo infatti notato che nelle case danneggiate, tutte di antica costruzione, erano state impiegate malte pochissimo coerenti, e che spesso i muri maestri erano sugli spigoli solamente accostati. Edifici moderni di una certa importanza e costruzioni in mattoni non hanno subito danni apprezzabili. Abbiamo voluto insistere su questo fatto per ribadire quanto è ormai accettato da molti e cioè che il criterio di valutazione dell'entità di una scossa in base ai danni subiti dagli edifici, è quanto mai aleatorio, se non intervengono a suffragarlo dati strumentali obiettivi come la magnitudo, la frequenza e l'ampiezza degli spostamenti del suolo, o almeno un'accurata indagine sulle norme costruttive che si sono seguite.

Data la scarsità del materiale a disposizione non abljiamo potuto eseguire alcuna indagine sulla natura fisica della scossa allo ipocentro, anche perché nelle registrazioni a disposizione, gli inizi sono quanto mai delooli se si escludono le stazioni di Roma e di Puy de Dôme.

Lo studio microsismico del terremoto è stato eseguito utilizzando i sismogrammi di 14 stazioni complessivamente, cioè le uniche clie abbiano ottenuto delle registrazioni sufficientemente intense; di queste solo in 8 si riscontra un inizio chiaro ed inequivocabile. Data la esiguità del materiale disponibile, ne segue che i risultati ottenuti dovranno assumersi con cautela, e quindi utilizzabili come eventuale punto di partenza per successivi studi necessari per l'accrescimento della conoscenza della struttura del Tirreno centro-settentrionale, zona finora poco nota sismicamente.

Determinazione dell'epicentro. - In base ai dati macrosismici e all'andamento delle isosiste, abljiamo assunto come epicentro provvisorio quello indicato da Strasburgo e precisamente

$$
\tau_{0}=41^{\circ}, 1 \mathrm{~N} \quad ; \quad \gamma_{0}=8^{\circ}, 9 \mathrm{E}
$$

assegnando provvisoriamente una profondità ipocentrale di $5 \mathrm{Km}$. Al)biamo quindi eseguito vari tentativi per la determinazione del tempo origine provvisorio utilizzando successivamente le dromocrone calcolate da Caloi (Cansiglio e Appennino), Marcelli-Di Filippo (Gran Sasso), Mohorovicic, Jeffreys; in tutti i casi si è riscontrata una notevole discordanza tra il tempo origine calcolato con le stazioni del Centro 
Europa e quello ottenuto dalle stazioni italiane. In particolare per Roma, che sulla componente verticale Wilip presenta un "impetus" si ha una discordanza di 4 s. Detto scarto, che si mantiene anche per la stazione di Prato, (le altre stazioni della rete italiana hanno inizi piuttosto incerti) non potendo essere imputato ad una erronea valutazione del tempo assoluto, può solo essere giustificato o da una velocità apparente diversa delle onde sismiche nelle due direzioni Sardegna-Europa Centrale e Sardegna-Italia, o da una erronea valutazione della posizione dell'epicentro provvisorio.

Onde scartare una delle due ipotesi, abbiamo calcolato la posizione dell'epicentro utilizzando dei dati di tutte le 8 stazioni a disposizione, seguendo il metodo che si vale delle differenze dei tempi di registrazione delle onde trasversali e longitudinali già applicato con successo da Caloi e successivamente da altri sperimentatori. Come è noto, tale metodo prescinde dalla conoscenza della velocità di propagazione e fornisce oltre alle coordinate dell'epicentro, la profonditi ipocentrale e il rapporto

$$
K=\frac{v_{1} v_{\mathrm{t}}}{v_{1}-v_{1}}
$$

I calcoli eseguiti per 3 approssimazioni successive hanno fornito:

$$
\begin{array}{lll}
\uparrow_{\mathrm{o}}=41^{\circ} 36^{\prime}, 8+5^{\prime}, 9 & ; & \lambda_{\mathrm{o}}=8^{\mathrm{n}} 24^{\prime} .4+0^{\prime}, 5 \\
h_{\mathrm{o}}=13+0,4 \mathrm{Km} & ; \quad K=9,2+0,4
\end{array}
$$

l'epicentro cadrebbe quindi al largo del ğolfo di Vallinco (Corsica), in evidente contrasto con l'andamento delle isosiste e quindi con la posizione dell'epicentro macrosismico. Resta quindi confermala l'ipotesi che la velocità di propagazione delle onde è diversa nelle due dire. zioni considerate; si è deciso quindi di scartare le stazioni Italiane ed eseguire la determinazione dell'epicentro utilizzando delle 6 stazion dell'Europa Centrale e precisamente Zurigo, Stoccarda, Strasburgo, Basilea, Neuchâtel, Puy de Dôme. Utilizzando del metodo di CaloiPeronaci che si vale del tempo di registrazione della fase iniziale e dei tempi di tragitto della medesima, e usando delle dromocrone di Mohorovicic si è ottenuto con 2 approssimazioni successive

$$
\begin{aligned}
& \varphi_{\mathrm{o}}=41^{\circ} 29^{\prime}, 4+31^{\prime}, 8 \\
& \lambda_{\mathrm{o}}=8^{\circ} 24^{\prime}, 2+10^{\prime}, 0 \\
& \boldsymbol{h}_{\mathrm{o}}=28+13 \mathrm{Km} \\
& H_{\mathrm{o}}=52^{\mathrm{m}} 20^{\mathrm{s}}, 8+1^{\mathrm{s}}, 5 .
\end{aligned}
$$


Ad analogo risultato si è giunti anche impiegando le dromocrone calcolate da Marcelli-Di Filippo per il terremoto del Gran Sasso.

$$
\begin{aligned}
& \varphi_{\mathrm{o}}=41^{\circ} 29^{\prime}, 2+30^{\prime} \\
& \lambda_{\mathrm{o}}=8^{\circ} 28^{\prime}, 2+8^{\prime} \\
& h_{\mathrm{o}}=20+10 \mathrm{Km} \\
& H_{\mathrm{o}}=52^{\mathrm{m}} 17^{\mathrm{s}, 8} 1^{\mathrm{s}, 2} .
\end{aligned}
$$

In entrambi i casi il risultato è sempre in notevole contrasto con i dati macrosismici, per cui abbiamo ritenuto che le dromocrone usate non rispecchiassero l'effettivo andamento della velocità delle onde sismiche nel tragitto Sardegna-Europa Centrale. Usando allora dei dati relativi all'epicentro macrosismico abbiamo provveduto a calcolare una dromocrona provvisoria delle $P_{n}$, utilizzando delle 6 stazioni suddette, si è ottenuto dall'applicazione del metodo dei minimi quadrati l'equazione:

$$
t=\frac{\Delta}{7,94 \pm 0,07}+8,74+0,7
$$

La velocita di propagazione delle $P_{n}$ si aggira quindi per il tragitto in questione sugli $8 \mathrm{~km}$ /sec, inferiore a quello trovato per il terremoto del Gran Sasso che è di $8,19 \mathrm{~km} / \mathrm{sec}$.

Abbiamo quindi applicato il metodo suaccennato utilizzando delle 2 dromocrone calcolate da Caloi per il Terremoto del Cansiglio $\left(h_{0}=17 \mathrm{~km}\right)$ e per quello dell'Appennino losco-romagnolo ( $h_{0}=5$ $\mathrm{km}$ ca.) che forniscono per le $P_{\mathrm{n}}$ la velocità di 8,0 e $7,86 \mathrm{~km} / \mathrm{sec}$ rispettivamente (si tenga presente che la dromocrona per il $2^{\prime}$ terremoto fu calcolata utilizzando esclusivamente stazioni dell'Europa Centrale). Il risultato dei calcoli spinto alla terza approssimazione ha fornito:

$$
\begin{aligned}
& r_{0}=41^{\circ} 3^{\prime}, 6 \pm 5^{\prime}, 9 \\
& \lambda_{0}=8^{\prime \prime} 41^{\prime}, 4 \pm 0^{\prime}, 5 \\
& h_{0}=13 \pm 5 \mathrm{~km} \\
& H_{0}=52^{\mathrm{m}} 12^{\mathrm{s}}, 5 \div 00^{\mathrm{s}, 7}
\end{aligned}
$$

in ottimo accordo con l'epicentro macrosismico. Naturalmente sarebbe stato del massimo interesse riprocedere alla determinazione con i dati delle stazioni italiane, ma per le ragioni su esposte ciò non è possibile. 
Dromocrone e velocità apparenti. - Abbiamo calcolate le dromocrone relative alle varie fasi; lo spoglio dei sismogrammi ¿̀ riassunto nella scguente tabella:

\begin{tabular}{|c|c|c|c|c|c|c|c|c|c|}
\hline Stazioni & Distanza & $I_{\mathrm{n}}$ & $P^{*}$ & & $s_{11}$ & & $S^{*}$ & & $M$ \\
\hline Roma & $331 \mathrm{~km}$ & $52^{m} 57^{\mathrm{s}}, 4$ & & & 131 s,0 & & & $5 t^{11}$ & $104^{\mathrm{s}}$ \\
\hline Firenze Arc. & 36.4 & & & & 37,8 & & e. & & 16,7 \\
\hline Prato & 370 & & & & 41.0 & & & & 17 \\
\hline Bologna & 437 & & & & 55,0 & & & & 39.8 \\
\hline Pavia & 459 & & & & 00,3 & & & & 49.5 \\
\hline Sal & 526 & & & & 16,2 & & & 5.5 & 08 \\
\hline Neuchàtel & 673 & 5314,5 & $4{ }^{n} 01 \times, 7$ & & 53,0 & $55^{m} 0.4^{s}, 0$ & $55^{\prime \prime 1} 77^{*}, 0$ & 56 & 07,9 \\
\hline Puy de Dôme & 689 & 46,9 & $0+, 2$ & & 56,5 & 06,5 & 20,0 & & 15 \\
\hline Zurigo & 700 & 48,7 & 06,7 & 55 & 00,6 & 10,9 & 24,6 & & 20 \\
\hline Basilea & 725 & 51,3 & & & 06,2 & 16.2 & 30,5 & & 28 \\
\hline & 838 & $5+\quad 06,0$ & & & 31,3 & 44,0 & 39,5 & 57 & 09.5 \\
\hline Stoccarda & 856 & 08.0 & 29,5 & & 35.0 & 50,2 & $56 \quad 06,7$ & & 14 \\
\hline
\end{tabular}

Per quanto riguarda le $P_{\mathrm{n}}$ si è potuto calcolare la dromocrona solo per le stazioni del Centro Europa e si è ottenuta l'equazione

$$
t=\frac{\Delta}{7,84-0,11}+6,5-0,7
$$

La velocità delle $P_{n}$ coincide con il valore ricavato da Caloi per l'Appennino tosco-romagnolo; per quanto riguarda la direzione Sardegna-Italia facciamo solo notare che nella stazione di Roma si riscontra nel tempo di registrazione una differenza di - $3^{\mathrm{s}}, 5$.

La fase $S_{n}$ ì risultata chiara in tutti $i$ sismogrammi: ne seque che abbiamo potuto calcolare le dromocrone nelle due direzioni suddette ottenendo per le stazioni oltre $\mathrm{Al}_{\text {pe }}$

$$
t=\frac{\Delta}{4,38+0,07}+7,44+1,0
$$

e per le italiane

$$
t=\frac{\Delta}{4,32 \div 0,09}+1.81 \div 1.5
$$

Tenuto conto degli errori, possiamo quindi concludere che pur essendo la velocita delle trasversali uguale nelle due direzioni, per le stazioni italiane si riscontra un anticipo di registrazione dell'ordine di 5 sec. 
Tale anticipo Irova il corrispondente per la longitudinale nella slazione di Roma che, come si è visto, riceve 3,5 sec prima del tempo calcolato.

Lna spiegrazione di questo fatto potrebloc esserc ricercata nel diverso spesso. re dello strato del granito nelle due direzioni in esane; infatti is accertato the tale strato sulisec un graduale assottigliamento quando si pas. si dall'Europa Centrale all'Italia. Naturalmente a causa dello scarso materiale a disposizione siamo costretti a limitarci a questa considerazione cli carattere qualitativo $c$ rimandare ad alıra occasione la possibilisa di un'inda. gine di natura guantitativa sulla strutura della crosta nella zona del Tirreno cen-

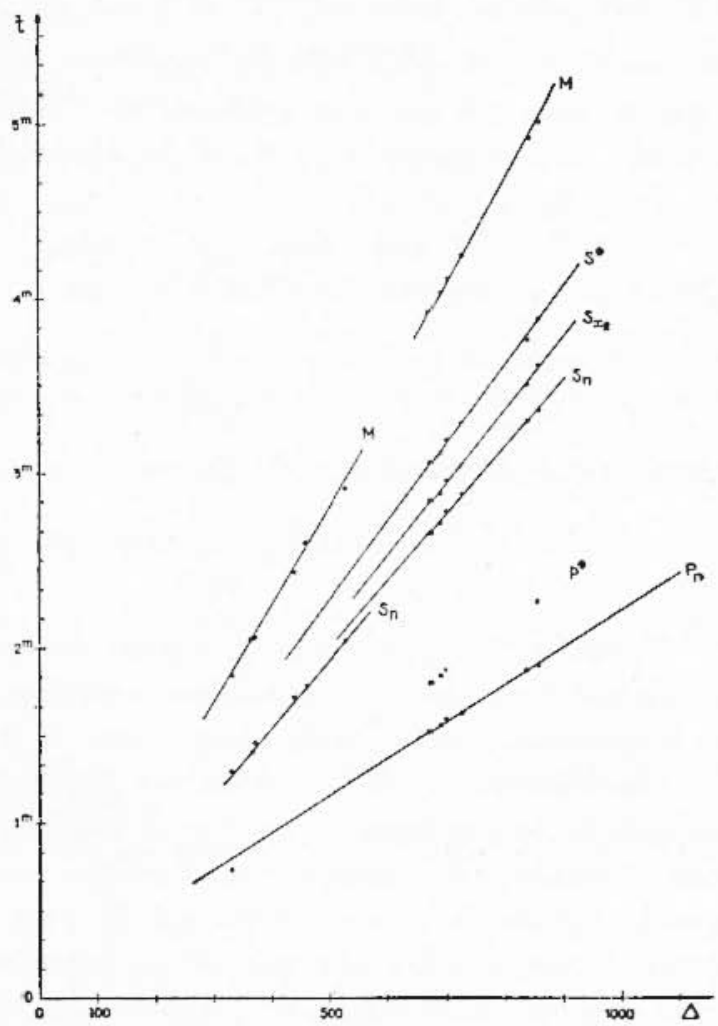

Fin. 2 trale.

Le $S^{*}$ sono state registrate con gran chiarcza solo nelle stazioni del Centro Europa o i calcoli hanno condotto all'equazione

$$
-\frac{\Delta}{3,74+0,09}+4,2: 0.8
$$

Come si osserva nella tabella la longitudinale corrispondente è rilevabile solo in 4 stazioni. La velocità delle $S^{*}$ coincide con il valore trovato da Gutenlycro per la Germania Meridionale e da Jefreys come media di vari terrenoti.

Nello spoglio dei sismogrammi si è riscontrata la presenza di una 
fase particolarmente evidente con periodo più ampio delle $S_{n}$ : la dromocrona calcolata con i tempi di 6 stazioni $\grave{e}$

$$
t=\frac{\Delta}{4,00 \div 0,08}+2,8 \div 0,7
$$

in base al valore della velocità apparente essa quindi risulta vicina a quelle onde già poste in evidenza da Caloi nel terremoto dell'Appennino tosco-romagnolo $(4,01)$ e da Marcelli-Di Filippo in quello del Gran Sasso $(4,04)$.

Passando alle onde della fase massima $(M)$ i calcoli ci hanno portato per le stazioni del Centro Europa a

$$
t=\frac{\Delta}{2,78}-5,65
$$

mentre per le italiane si è ottenuto

$$
t=\frac{\Delta}{3,0}+2,8
$$

Naturalmente trattasi per le ragrioni suddette, di valori puramente indicativi; ciò non toglie però clse si noti un aumento della velociti di propagazione nei tragitti interessanti il Tirreno Centrale.

Concludendo abbiamo notato un anticipo sui tempi di registrazione delle longitudinali e trasversali nei tragitti interessanti il Tirreno Centrale; tale aumento risulterebbe più o meno costante per tutte le distanze e si aggirereblue sui $5^{\text {s }}$ circa per le trasversali; riteniamo di conseguenza che esso possa imputarsi ad una diminuzione dello spessore dello strato del granito nella direzione in questione; tale ipotesi restereblue suffragata dal valore più elevato della velociti di propagazione delle onde della fase $M$ nella stessa direzione, se si tiene presente che l'influenza delle stratificazioni superficiali risulterebbe notevolmente diminuita.

Roma - Istituto Nazionale di Geofisica - Novembre 1953.

\section{RIASSUNTO}

Viene studiato il terremoto Sardo del 1.3 novembre 1948. Si pone in evidenza un diverso comportamento nella propagazione delle onde elastiche nella direzione Sardegna-Italia rispetto ai tragitti interessanti le stazioni dell'Europa Centrale. 


\section{SUMMARY}

The Sardinian earthquake of November 1.3, 1948 has been studied. It is shoun that the elastic uave in the direction Sardiniu-Italy has a different behavior with respect to the tracings of interest to the stations of Central Europe.

\section{BIBLIOGRAFIA}

Calor l'., Determinazione delle coordinate epicentrali di un terremolo ad origine vicina con i tempi delle onde longitudinali e trusversali dirette. "La ricerca scientifica ), 1911 , pag. 431.

Calor-Penoxaci, $1 l$ terremoto del Turkestan. "Annali di Geofisicia", vol. I, n. 2 (19.48).

Calor P., Tempi di tragillo per terremoli ad origine vicina. "La ricerca scientifica », 1939, pag. 388.

Calor P., Carutteristiche sismiche dell Appennino tosco-romagnolo, "La ricerca scientifica ", 1940, pag. 218.

Di Filmpo-Mancelet, Dromocrone per terremoti vicini e velocitì delle onde nellltalia Centrale. "Annali di Geofisira ", vol. V, n. 2 (1952).

C.aloo P., Struttura geologico-sismica dellFuropa centro-meridionale ecc... "Annali di Geofisica ", vol. V, n. 1, 11952).

Caror P., Attivitì sismica in lalin nel decennio 1930-1939. Le Monnier, Firenze, (19.42). 